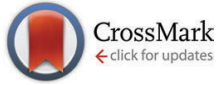

Cite this: Phys. Chem. Chem. Phys., $2016,18,22731$

DOI: $10.1039 / c 6 c p 90188 c$

\section{Correction: Photochemical etiology of promising ancestors of the RNA nucleobases}

\author{
M. M. Brister, M. Pollum and C. E. Crespo-Hernández* \\ Correction for 'Photochemical etiology of promising ancestors of the RNA nucleobases' by M. M. Brister et al., \\ Phys. Chem. Chem. Phys., 2016, DOI: 10.1039/c6cp00639f.
}

www.rsc.org/pccp

The authors wish to update the Electronic Supplementary Information (ESI) with the revised version that is included with this correction. See DOI: 10.1039/c6cp00639f. The energetic ordering reported in Table S1 of the originally-submitted ESI for the 16 tautomers of barbituric acid (BA) was tabulated incorrectly. The original Table S1 reported the enol(C6) tautomer of BA as the lowest-energy tautomer in water instead of the tri-keto tautomer. Hence, Table S1 and some of the discussion of the supplementary results in the ESI have been revised. In addition, we now report and discuss briefly new calculations for the five lowest-energy tautomers of BA complexed with three water molecules in the revised ESI.

The impact the revision has on the main text of the article is that any reference to 'the enol(C6) tautomer' should be replaced by the phrase 'an enol tautomer'. In addition, the phrase "Density functional (DFT) calculations of 16 different tautomers of BA show that the enol(C6) tautomer is the predominant species in neutral aqueous solution" should be replaced by "Density functional (DFT) calculations of 16 different tautomers of BA, including calculations for five BA tautomers complexed with three water molecules, support the idea that an enol tautomer is the predominant species in neutral aqueous solution". Fig. S2 and Table S2 referenced in the main text of the article for the enol(C6) tautomer have been removed from the revised ESI, as the results are no longer relevant to the discussion. Fig. S5, S6, S7 and S8 referenced in the main text of the article are now Fig. S6, S7, S8, and S9, respectively, and Table S4 is now Table S9 in the revised ESI.

These changes do not affect the discussion or the conclusions reported in the paper.

\title{
Acknowledgements
}

The authors would like to thank Ms Shahila Muhammed and Dr Sudha Rajamani from the Indian Institute of Science Education and Research at Pune, India for calling attention to this issue. The authors apologize for the inconvenience.

The Royal Society of Chemistry apologises for these errors and any consequent inconvenience to authors and readers. 